\title{
Mesures des débits par méthode ultrasonore
}

\author{
F. Multon \\ ULTRAFLUX
}

Les débitmètres à ultrasons ne sont apparus sur le marché sous forme réellement industrielle que vers 1970 . Bien que le principe en soit connu depuis fort longtemps, la mise en application de ce procédé s'est heurtée à l'insuffisance des performances des moyens électroniques de l'époque.

Depuis, l'apparition de l'électronique intégrée et des microprocesseurs a permis d'aboutir à des réalisations industrielles fiables et précises.

\section{Principe de mesure de vitesse par différence de temps de transit}

Parmi les différents procédés électroniques utilisés, effet Doppler, mesure de phase, singaround, intercorrélation, seule la mesure de la différence des temps de transit permet de réaliser une véritable intégration du profil des vitesses et conduit par conséquent à une meilleure précision.

Si on appelle $A$ et $B$ les deux sondes émettricesréceptrices (fig. I), $V$ la vitesse moyenne d'écoulement, $L$ la longueur du trajet acoustique et $C$ la vitesse de propagation du son dans le fluide au repos, les temps de propagation des impulsions ultrasonores de $A$ vers $B$ et de $B$ vers A s'écrivent :

$$
\begin{aligned}
T_{A B} & =\frac{L}{C+v \cos \theta} \\
T_{B A} & =\frac{L}{C-v \cos \theta}
\end{aligned}
$$

d'où l'on tire l'expression de la vitesse moyenne le long de la ligne $A B$. La vitesse ainsi mesurée représente l'intégrale du profil des vitesses dans le plan diamétral.

$$
\begin{gathered}
\frac{1}{T_{A B}}-\frac{1}{T_{B A}}=\frac{2 v \cos \theta}{L}=\frac{2 V D}{L^{2}} \\
V=\frac{L^{2}}{2 D}\left(\frac{1}{T_{A B}}-\frac{1}{T_{B A}}\right) .
\end{gathered}
$$

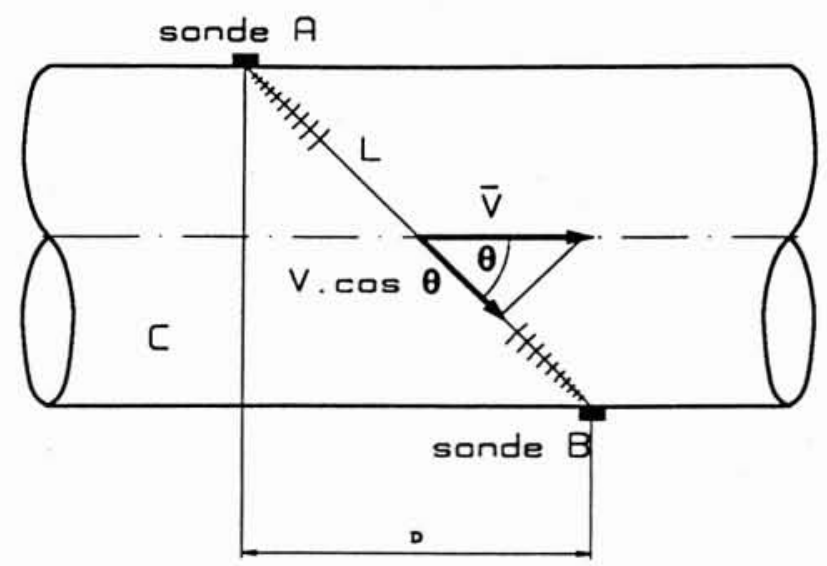

1. Mesure de vitesse par différence de temps de transit.

\section{Calcul du débit à partir de la vitesse mesurée}

Dans les conduites circulaires et en régime stabilisé, deux régimes d'écoulements différents peuvent prendre naissance, le régime laminaire pour des nombres de Reynolds inférieurs à 2800 , le régime turbulent pour les nombres de Reynolds supérieurs à 2800 .

On a coutume d'appeler coefficient hydraulique $K_{H}$ le rapport de la vitesse mesurée $V_{\text {us }}$ à la vitesse moyenne vraie obtenue en intégrant le profil de vitesse dans toute la section de mesure.

La connaissance de l'expression mathématique du profil de vitesse permet le calcul de ce coefficient.

En régime laminaire (fig. 2 ) :

$$
\begin{aligned}
V(y) & =V_{0}\left(1-\frac{y^{2}}{R^{2}}\right) \\
K_{I I} & =1,33 .
\end{aligned}
$$

\section{Flow measurement using ultrasonic method}

Ultrasonic flow measurement methods have been widely used in industry since the 70's.

Progress in the domain of electronic components performances lead to more precision concerning speed and flow measures, but it is necessary to master geometrical parameters of the establishment and the choice of the site.

The possibility of installing sensors on a loaded pipe and the use of external sensors make this method economically interesting for large pipes. 


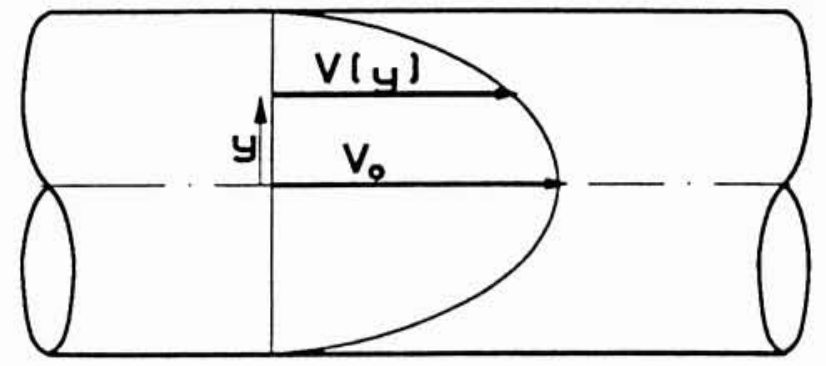

2. Calcul du coefficient $K_{H}$ en régime laminaire.

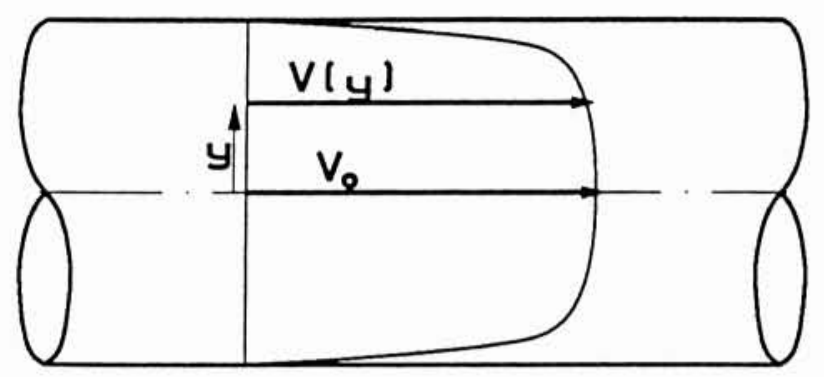

3. Calcul du coefficient $K_{H}$ en régime turbulent.

En régime turbulent (fig, 3), ce coefficient est une fonction du nombre de Reynolds.

$$
V(y)=V_{0}\left(1-\frac{y}{R}\right)^{\frac{1}{1}}
$$

avec :

$$
\begin{aligned}
\frac{1}{n} & =0,250-0,0231 \mathrm{~g} \mathrm{Re} \\
K_{/ \prime} & =F(\operatorname{Re}) .
\end{aligned}
$$

Les résultats du calcul complétés par de nombreux résultats expérimentaux conduisent à l'établissement du réseau de courbes représenté sur la figure 4 ci-après.

On s'aperçoit que, pour une conduite déterminée et pour une large dynamique de vitesse; ce coefficient évolue peu. Il est donc possible de calculer la vitesse moyenne vraie à partir de la mesure de la vitesse moyenne dans le plan diamétral.

$$
V=V_{u s} \cdot \frac{1}{K_{H}}
$$

d'où le calcul du débit :

$$
Q=\frac{\pi \phi^{2}}{4} \cdot \frac{1}{K_{H}} \cdot \frac{L^{2}}{2 d}\left(\frac{1}{T_{A B}}-\frac{1}{T_{B A}}\right) .
$$

Ce calcul n'est évidemment valable que si l'hypothèse faite sur l'expression mathématique du profil des vitesses est vérifiée, ce qui nécessite le respect de longueurs droites en amont (10 à 15 D) et en aval (3 à 5 D) du point de mesure.

Les courbes de la figure 4 sont établies pour une rugosité typique de quelques dixièmes de $\mathrm{mm}$.

On comprend aisément que l'écoulement dans la couche limite est fortement influencé par la rugosité. L'influence de cette perturbation est d'autant plus importante que le diamètre de la conduite est faible.

Certains hydrauliciens, et en particulier COLEBROOK, ont établi des lois qui prennent en compte la rugosité et dont l'application est simple, à condition toutefois de définir et de mesurer la rugosité...

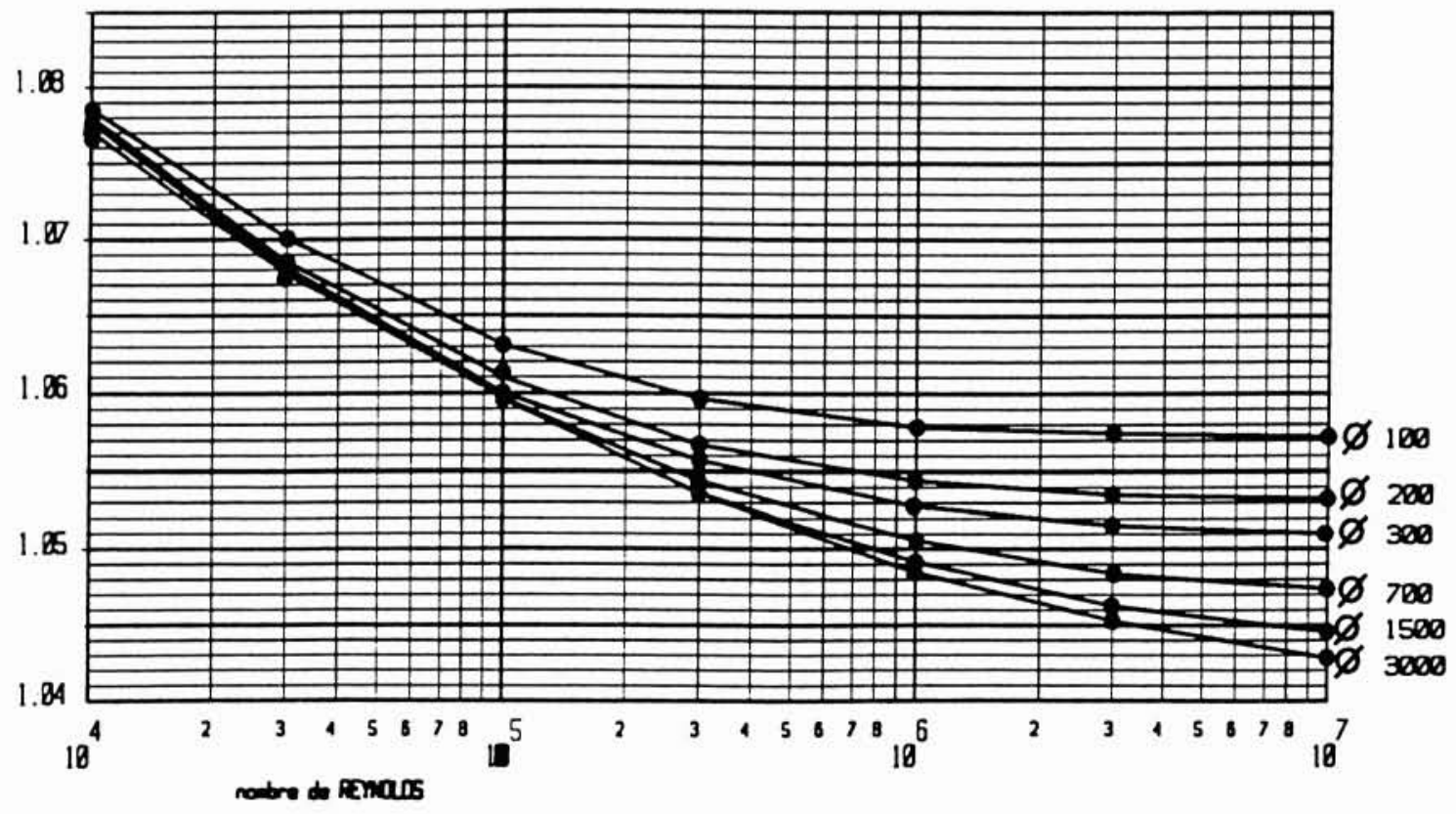

4. Courbes de $K_{h}$ en fonction du nombre de Reynolds. 


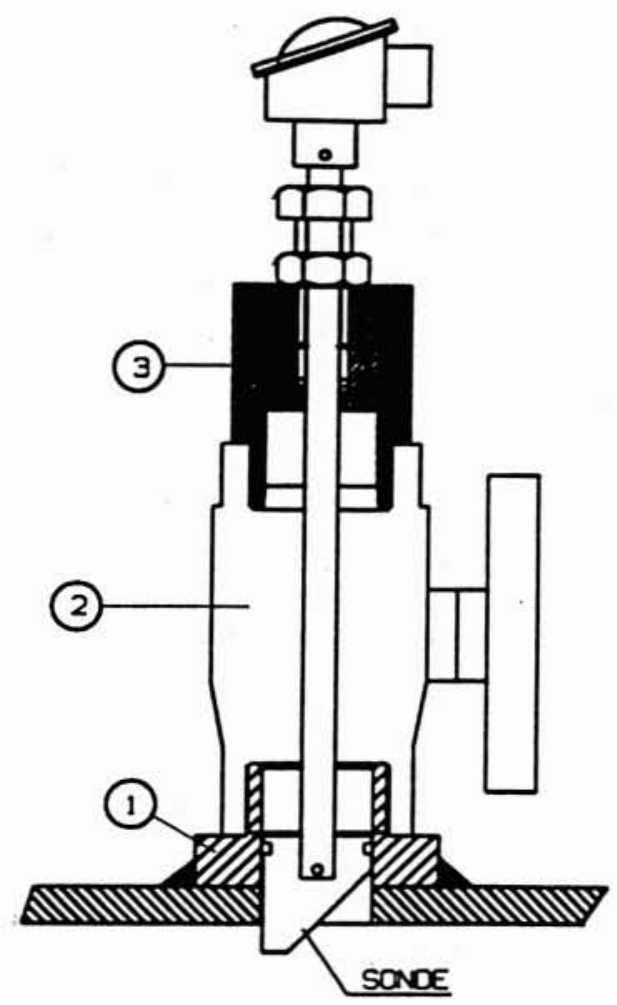

5. Disposition générale de capteurs intrusifs à insertion.

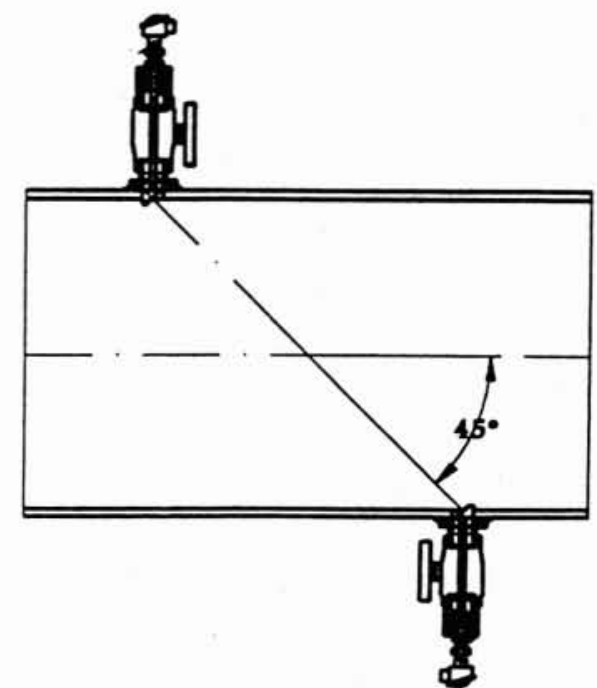

\subsection{Sondes extérieures}

La faculté que possèdent les ultrasons à traverser les milieux élastiques permet d'effectuer des mesures de débit en appliquant les sondes sur la paroi extérieure des canalisations.

Un matériau de couplage permettant un meilleur transfert d'énergie doit être placé entre la sonde et la tuyauterie. Cette technique est utilisable sur les conduites en acier, Inox, fonte revêtue ou non, plastique, PVC, fibro-ciment.

Les capteurs seront placés suivant le mode direct (fig. 6) pour les conduites de diamètre supérieur à $500 \mathrm{~mm}$ et en mode reflex sur des conduites de diamètre inférieur ou si la qualité de la conduite (fort entartrage par exemple) ne permet pas une bonne réflexion sur la paroi opposée. Le mode Reflex (fig. 7) doit toujours être préféré au mode Direct.

La position des capteurs dépend du diamètre, de l'épaisseur et de la nature de la tuyauterie et de celle du liquide. Elle est calculée par l'appareil.

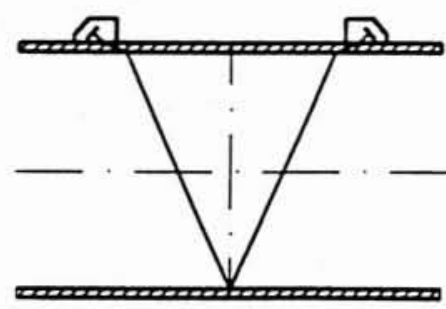

MOOE REFLX

7. Placement des capteurs en mode Reflex. 


\subsection{Etalonnage - Précision}

La formule qui permet le calcul du débit :

$$
Q=\frac{\pi \phi^{2}}{4} \cdot \frac{1}{K_{h}} \cdot \frac{L^{2}}{2 D}\left(\frac{1}{T_{A B}}-\frac{1}{T_{B A}}\right) .
$$

montre qu'il suffit de mesurer avec une précision suffisante les temps de propagation $T_{A B}$ et $T_{B A}$ et de connaître les grandeurs géométriques $\Phi, L, D$, ainsi que la valeur du coefficient hydraulique $K_{h}$ pour obtenir la valeur exacte du débit.

L'examen de la formule précédente montre clairement l'incidence des incertitudes sur $\Phi, L, D$ sur le calcul du débit $Q$. Il convient donc d'apporter le plus grand soin à leur mesure et de positionner les sondes correctement.

Dans le cas d'une sonde intrusive trop enfoncée, outre les turbulences qu'elle crée, les grandeurs $L$ et $D$ sont celles figurant sur la figure 8 .
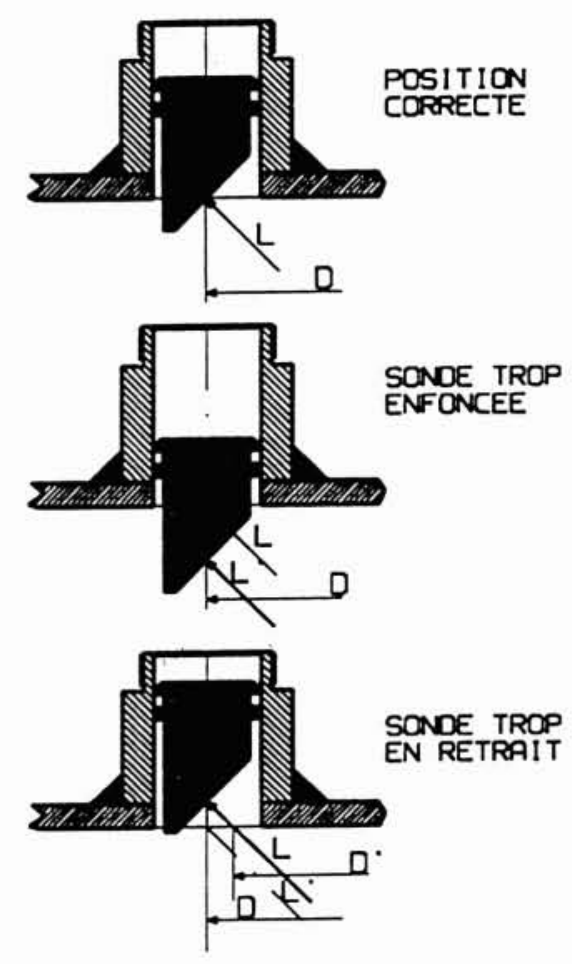

8. Grandeurs $L$ et $D$ suivant la position de la sonde.

La longueur $L$ paramétrée étant plus grande que la longueur réelle, l'erreur ainsi introduite conduit à un surcomptage aggravé par le fait que l'appareil ne tient plus compte de la couche limite.

Dans le cas d'une sonde en retrait, la longueur $L$ paramètrée est plus petite que la réalité d'où un sous-comptage aggravé cette fois par le fait que la distance axiale $D$ paramètrée est plus grande que la distance $D$ réelle.

La figure 9 montre un exemple de vérification de la précision d'un tel étalonnage. Il a été réalisé dans le Laboratoire d'essais de la SAGEP sur une conduite de $500 \mathrm{~mm}$ de diamètre avec des sondes intrusives. Les précisions y sont calculées en \% de la valeur mesurée et non en $\%$ de la pleine échelle.

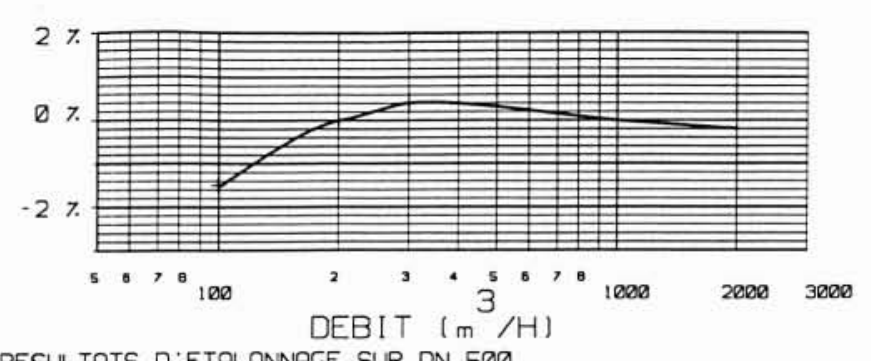

RESULTATS D'ETALONNAGE SUR DN 500

LABORATOIRE DES COMPTEURS DE LA SAGEP le $11 / 03 / 82$

9. Exemple de vérification de la précision d'un étalonnage.

L'altération de la forme des signaux acoustiques provoquée par la traversée des parois, conduit à une précision légèrement moins bonne ( 2 à $3 \%$ ) lorsqu'on utilise des sondes extérieures à la canalisation.

Ces résultats obtenus, après paramétrage et contrôle géométrique soigneux, confirment qu'il n'est pas nécessaire de réaliser un étalonnage hydraulique sur ces appareils.

\section{Débitmètres pour conduites pleines}

La Société Ultraflux a conçu les débitmètres numériques UF 321 pour les installations fixes et UF $321 \mathrm{~T}$ en version portable.
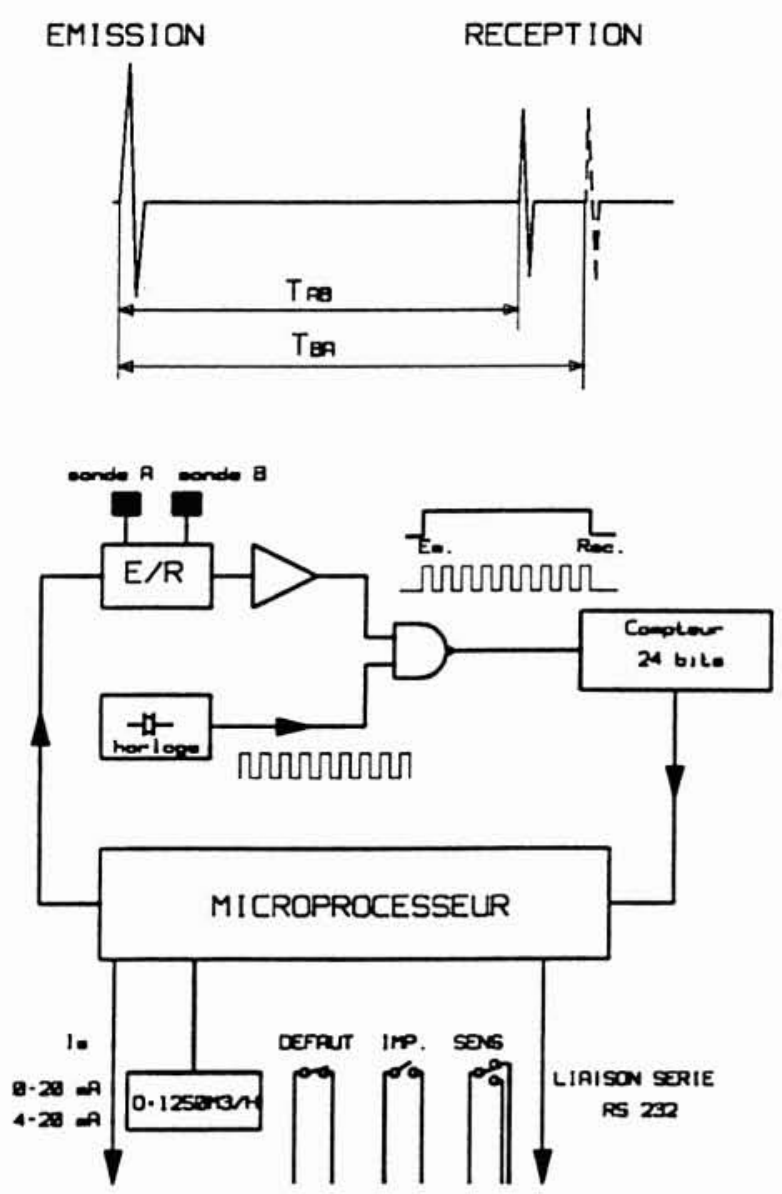

10. Schéma de fonctionnement de l'UF 321. 
Construits autour d'un microprocesseur, ils fonctionnent suivant le schéma de la figure 10. Le microprocesseur gère les séquences d'émission-réception amont-aval et avalamont, et mesure avec une résolution de 1 nanoseconde $\left(10^{-9} \mathrm{~s}\right)$ les temps $T_{A B}$ et $T_{B A}$. Il calcule ensuite le débit à l'aide des paramètres $\Phi, L, D$ entrés directement au clavier lors du paramétrage de l'appareil.

Le débit est indiqué sur un afficheur LCD et une sortie analogique (0-20 ou 4-20 mA) est l'image du débit.

L'appareil fonctionne dans les deux sens d'écoulement. Il contrôle en permanence la validité des mesures et la qualité de la transmission acoustique et délivre une alarme en cas de défaut.

Une option lui permet d'effectuer du comptage. Les informations du débit et du volume sont disponibles sur une liaison série RS 232. Cette option permet également le choix du langage de programmation (Français, Anglais, Italien, Portugais, Allemand).

\section{Systèmes multicordes}

Lorsque les conditions hydrauliques sont mauvaises (absence de longueurs droites par exemple), l'hypothèse faite sur le profil des vitesses n'est plus vérifiée et il devient impossible de définir la valeur du coefficient hydraulique $K_{H}$.

Le problème est résolu par l'utilisation de systèmes multicordes de la série UF 2100 (fig. 11) qui permettent d'effectuer la mesure de la vitesse réelle existant en différents points de la veine liquide. Le calcul du débit s'effectue alors par intégration mathématique (Méthode de Gauss, Newton ou autre).

On obtient par cette méthode une précision de $+/-1 \%$ lorsque les conditions hydrauliques sont médiocres et de $+/-0,3 \%$ en profil stabilisé et dans une dynamique beaucoup plus grande (particulièrement vers les faibles vitesses).

Ces appareils peuvent également être utilisés en multiconduite et multicorde avec un maximum de 4 voies de mesures par appareil UF 2100 (fig. 12).

\section{Mesures de débit en conduites non pleines, canaux ouverts, rivière}

Dans les écoulements à surface libre, la seule mesure de niveau n'est pas suffisante pour la connaissance du débit (influence des conditions aval par exemple).

Dans ce cas, la mesure des vitesses et du niveau sont indispensables. Les appareils de la série UF 2100 sont conçus pour effectuer 4 mesures de vitesse et 1 mesure de niveau. Ils peuvent également recevoir un signal extérieur de niveau.

Les sondes sont généralement placées suivant la figure 13.

L'appareil est totalement paramétrable. Les éléments nécessaires à son réglage sont :

- les paramètres géométriques pour chaque paire de sondes $(h, L, D)$;

- la description géométrique de la section de mesure.

L'appareil mesure les vitesses et le niveau, puis calcule le débit global en effectuant la sommation des débits élémentaires par tranche horizontale de surface.

Les appareils de la série UF 2100 possèdent (fig. 14) :

- 1 clavier numérique permettant le paramétrage ;

- 1 afficheur LCD ;

- 5 sorties analogiques paramétrables qu'il est possible d'affecter à l'une quelconque des 60 grandeurs mesurées par l'appareil dont évidemment $V 1, V 2, V 3, V 4, H, Q$, Température ;
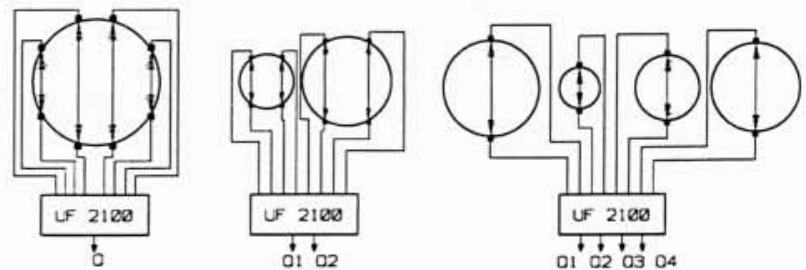

12. Utilisations en multiconduite et multicorde de I'UF 2100.

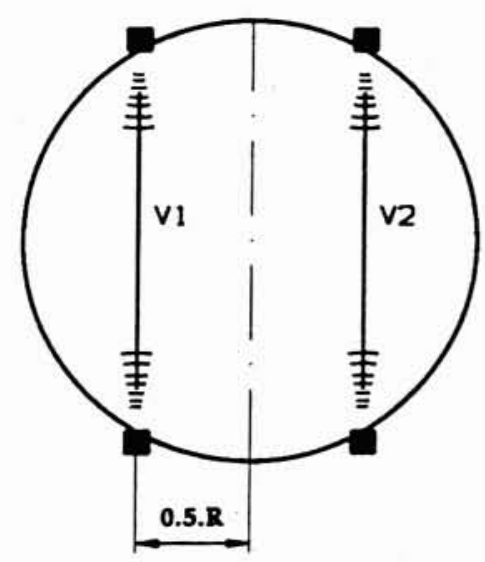

2 COROES RVEC INTEGRTIION PAR RETHOOE DE GAUS-JCOB! $0 \cdot 5.10 .500 \mathrm{~V} 1 \cdot 0.500 \mathrm{~V} 21$

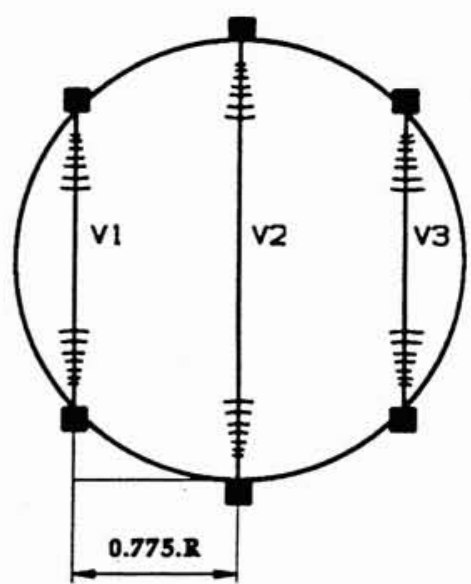

3 COROES AVEC INTEGPATION PAR TETHOOE DE GASS 0. S. 10.444 V1 0.555 V2・0.444 V31

11. Systèmes multicordes de la série UF 2100. 

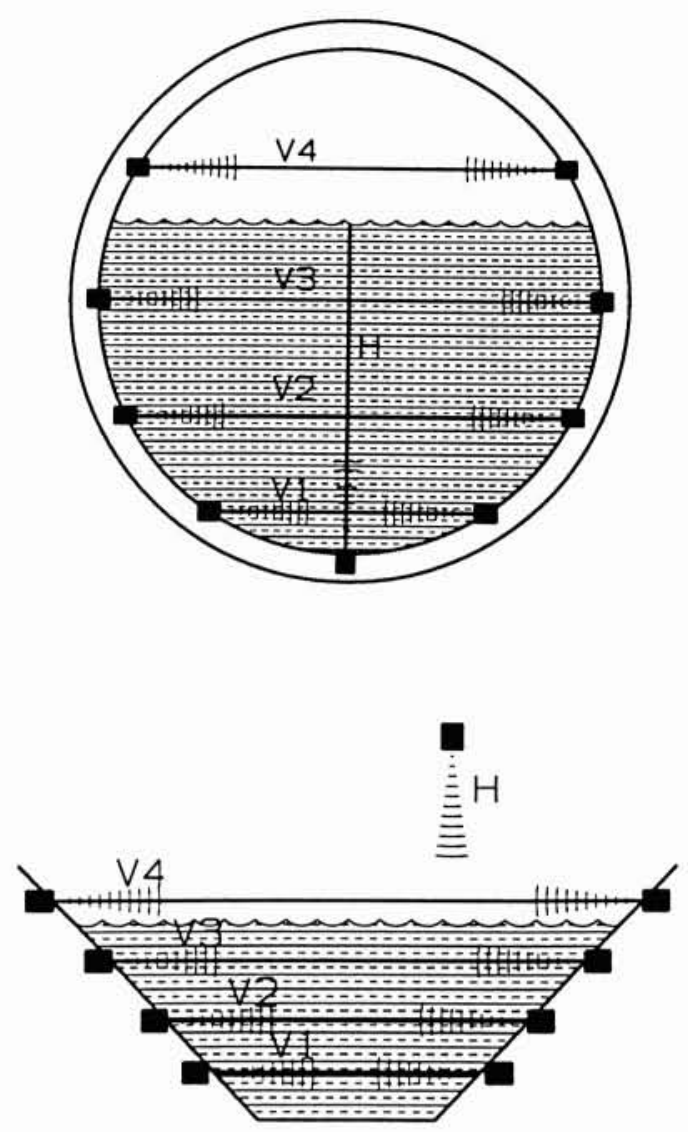

13. Placement des sondes pour une mesure en conduite non pleine.

- 1 mini-logger permettant d'enregistrer sous forme numérique une grandeur moyennée sur 1,10 minutes, 1 heure ou 1 journée :

- 1 liaison série RS 232;

- en option, on peut ajouter un logger de $64 \mathrm{k}$ qui permet le stockage des mesures pour traitement ultérieur sur ordinateur ;

- 1 logger de $0,7 \mathrm{~K}$ appelé logger de défaut qui enregistre tous les événements horodatés qui surviennent pendant le fonctionnement de l'appareil :

- défauts de mesure,

- pertes de transmission acoustique,

- coupures secteurs et remises sous tension,

- accès à la programmation.

L'appareil peut être raccordé via la liaison série :

- à un PC portable pour transfert des données ou téléchargement d'un nouvel étalonnage ;

- à une imprimante locale ;

— à un Modem pour interrogation à distance via le réseau téléphonique.

\section{Limitations et avantages des débitmètres à ultrasons}

\subsection{Limitations}

\subsection{Sondes}

De part la nature même des céramiques piézo-électriques utilisées dans les sondes, leur température d'utilisation est limitée à la température de Curie, température à laquelle elles perdent les propriétés piézo-électriques. Cette température est de l'ordre de $300{ }^{\circ} \mathrm{C}$. La technologie de construction (câbles, connecteur, matériaux) limite généralement leur emploi à $180^{\circ} \mathrm{C}$.

\subsubsection{Fluide}

Un choix convenable de la fréquence et de la dimension des sondes permet l'utilisation des débitmètres à ultrasons à temps de transit sur des fluides parfaitement propres, chargés (jusqu'à $10 \mathrm{~g} / \mathrm{l}$ ) et légèrement visqueux ([Lz] $200 \mathrm{cst}$ ).

Les problèmes de corrosion et de pression sont parfaitement résolus par l'usage de sondes extérieures.

\subsection{Avantages}

Les principaux avantages de la méthode ultrasonore sont : - sonde entièrement statique, sans pièces en mouvement ;

- mesure bidirectionnelle ;

- possibilité d'installation des sondes intrusives sur conduite existante sans interruption du débit ;

— possibilité d'étalonnage sur site ;

- contrôle permanent de la validité des mesures ;

- contrôle mobile des débits sur réseaux ;

— pas de limitation, ni de vitesse, ni de diamètre.

Les prix de ces matériels, particulièrement attractifs pour les conduites de grand diamètre, en font un outil très apprécié des spécialistes : de la distribution d'eau potable, de l'assainissement, de l'irrigation, de la gestion des canaux et rivières.

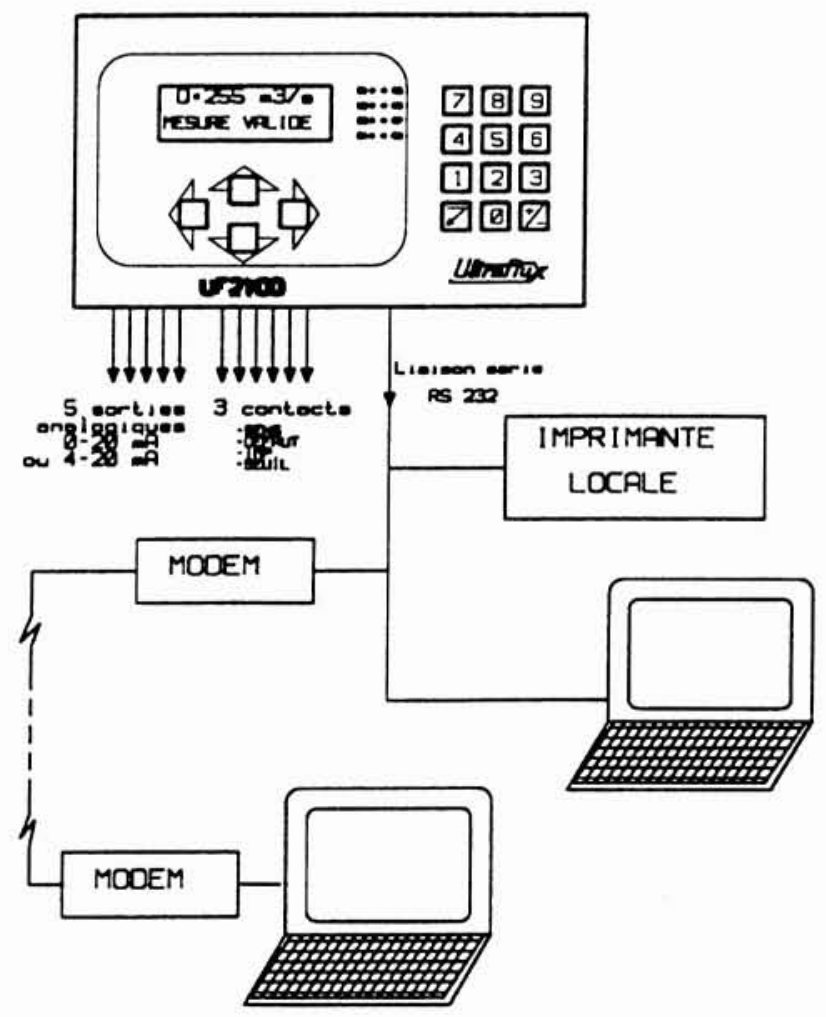

14. Schéma de l'UF 2100. 